\title{
CARACTERIZACIÓN FENOTÍPICA Y FUNCIONAL DE RIZOBIOS NODULADORES DE DOS ESPECIES DEL GÉNERO MACROPTILIUM
}

\author{
Toniutti, M. A. ${ }^{1}$ Fornasero, L.V. ${ }^{1}$ Trod, B.S. $^{1}$ \\ ZUBeR, N.E. ${ }^{1} \&$ CÓRDOBA, M.S. ${ }^{1}$
}

\begin{abstract}
RESUMEN
Macroptilium atropurpureum y Macroptilium bracteatum son leguminosas de interés forrajero valoradas por su capacidad de fijar nitrógeno atmosférico en relaciones simbióticas con rizobacterias. Aislados provenientes de nódulos de ambas especies del género Macroptilium fueron caracterizados fenotípicamente y a nivel funcional. Las diecinueve cepas estudiadas presentaron colonias pequeñas, translúcidas, poco gomosas y de crecimiento lento. En el análisis de agrupamiento, las cepas de referencia se diferenciaron claramente de los aislados en estudio, los cuales se agruparon formando cuatro conglomerados según la velocidad de crecimiento, tolerancia a temperaturas, niveles de $\mathrm{pH}$, concentraciones de sales y resistencia a diferentes antibióticos. Se identificaron siete aislados capaces de crecer en condiciones de altas temperaturas, alcalinidad y/o salinidad, los cuales tendrían un uso potencial como biofertilizantes.

Palabras Claves: Macroptilium artropurpureum; Macroptilium bracteatum; rizobios; fenotípica; tolerancia a factores abióticos.
\end{abstract}

\begin{abstract}
Functional and phenotypic characterization of nodular rhizobia from two species of macroptiliun genus.

Macroptilium atropurpureum y Macroptilium bracteatum are legumes of forage interest recognized for their ability to fix atmospheric nitrogen in symbiotic relationships with rhizobacteria. Isolates coming from nodules of both Macroptilium species were characterized functional and phenotypically. The nineteen strains studied showed small colonies which were translucent, a bit gummy and low growth. In the grouping test, the previous strains clearly differed from the isolates under investigation. These gathered, making four mixtures according to the speed of growth,
\end{abstract}

1.- Facultad de Ciencias Agrarias. Universidad Nacional del Litoral. Kreder 2805.

(3080) Esperanza, provincia de Santa Fe. Email: matoniut@fca.unl.edu.ar

Manuscrito recibido el 12 de diciembre de 2014 y aceptado para su publicación el 5 de agosto de 2015. 
M. A. Toniutti et al.

temperature tolerance, $\mathrm{pH}$ levels, salt concentration and resistance to different antibiotics. Seven isolates capable of growing under conditions of high temperature, alkalinity and / or salinity which would have a potential use as biofertilizers were identified.

Key Words: Macroptilium artropurpureum, Macroptilium bracteatum, rhizobia phenotypic tolerance to abiotic factors.

\section{INTRODUCCIÓN}

El empleo de leguminosas nativas asociadas a gramíneas constituye un recurso eficiente para mantener el suelo con buenos niveles de fertilidad debido al nitrógeno transferido a la gramínea proveniente de la fijación simbiótica entre la leguminosa y los rizobios asociados $(35,38,42)$ lo cual podría minimizar el uso de fertilizantes químicos generalmente costosos, de baja eficacia y alto impacto ambiental. Las especies de leguminosas nativas adaptadas a nuestras condiciones edafoclimáticas presentan un gran potencial de producción forrajera.

Entre estas especies se destacan las que pertenecen al género Macroptillum, tales como M. atropurpureum (MA) y M. bracteatum (MB), plantas herbáceas anuales o perennes y de ciclo primavero-estivo-otoñal $(11,30)$. MA es una especie nativa de América que crece hasta el sur de Brasil, utilizada como forrajera en ambientes subtropicales y tropicales, existiendo dos cultivares, "Siratro" y "Aztec" (6, 8,32).M. bracteatum es una especie nativa de América y en Argentina se la encuentra en las regiones fitogeográficas Paranaense, Yungas y Chaqueña $(23,33)$ de reconocido potencial forrajero, habiéndose liberado en Australia dos cultivares, "Cadarga" y "Juanita" $(13,21,30)$.

Estas leguminosas, al igual que la mayoría de las especies forrajeras perennes presentan un crecimiento inicial lento como limitante para su implantación $(5,10)$. Otro 106 de los factores determinantes de un establecimiento exitoso es una efectiva fijación biológica de nitrógeno a través de la simbiosis con rizobios noduladores $(2,5,40)$.

Las leguminosas presentan distintos grados de especificidad en relación al rango de especies de rizobios capaces de nodularlas. En las Fabaceas, sub-familia Papilionoideae se encuentran las plantas más promiscuas o capaces de nodular efectivamente con varias especies de rizobios (24).

Entre ellas se destaca por su promiscuidad el género Macroptilium $(22,34)$, siendo generalmente nodulado por Bradyrhizobium sp. aislado de diversas leguminosas, incluyendo especies de Vigna, Lupinus, Ornithopus, Cicer, Sesbania, Leucaena, Mimosa, Lablab, y Acacia (22) y por B. japonicum y B. elkanii, simbiontes de Glycine max (27). Asimismo, las plantas de Macroptilium también pueden ser noduladas por cepas de crecimiento rápido de los géneros Rhizobium y Ensinfer, que se hallan filogenéticamente distantes de Bradyrhizobium como E. melilotti (7), R. leguminosarum biovar trifolii, y $R$. leguminosarum bv. phaseoli (41).

Por otro lado, diversos factores ambientales como acidez del suelo, temperaturas extremas o salinidad, serían perjudiciales para la supervivencia y diversidad de las poblaciones de rizobios nativos y para su capacidad de establecer asociaciones simbióticas $(3,14)$. La caracterización fenotípica de los rizobios nativos adaptados a condiciones estresantes en los suelos consRevista FAVE - Ciencias Agrarias 14 (1) 2015 